\title{
CALT: Uma Ferramenta Automática para Cobrança do ICMS em Operações Interestaduais
}

\author{
Anthony E. A. Jatobá ${ }^{1,2}$, Douglas L. L. Moura ${ }^{2,3}$, Ivan C. Martins ${ }^{2}$, \\ Heitor S. Ramos ${ }^{2,3}$, André L. L. Aquino ${ }^{1,2}$ \\ ${ }^{1}$ Instituto de Computação - Universidade Federal de Alagoas (UFAL) \\ ${ }^{2}$ Laboratório de Computação Científica e Análise Numérica \\ Universidade Federal de Alagoas (UFAL) \\ ${ }^{3}$ Departamento de Ciência da Computação \\ Universidade Federal de Minas Gerais (UFMG) \\ \{anthonyjatoba, douglas.moura, ivan.martins, alla\}@laccan.ufal.br \\ ramoshedcc.ufmg.br
}

\begin{abstract}
Due to constant legislation changes and the Brazilian tax system's complexity, calculating the tax on a product is not a trivial task. For this reason, this paper presents a proposal for a tool for the automatic calculation of taxes charged in interstate transactions destined to the state of Alagoas. Based on clustering and classification techniques, the proposed work aims to learn the taxation patterns in previously calculated electronic invoices and, thus, provide a model capable of predicting the taxation of a product. In this way, the automatic calculation offers a straightforward manner to obtain the ICMS charges with an immeasurable improvement in the processing time of electronic invoices compared to manual calculation, which in a practical manner was not performed on all electronic invoices.
\end{abstract}

Resumo. Devido a mudanças constantes na legislação e a complexidade do sistema tributário brasileiro, determinar a tributação de um produto não é uma tarefa trivial. Com isso, este artigo apresenta uma proposta de ferramenta para o cálculo automático dos tributos devidos nas operações interestaduais destinadas ao estado de Alagoas. Mediante o uso técnicas de agrupamento e classificação, o trabalho proposto pretende aprender os padrões de tributação em notas fiscais previamente calculadas e, deste modo, fornecer um modelo capaz de predizer a tributação de um produto. Dessa forma, o cálculo automático oferece uma maneira facilitada de obter a cobrança do ICMS com uma melhora imensurável no tempo de processamento das notas eletrônicas em relação ao cálculo manual, que de forma prática não era realizado em todas as notas.

\section{Introdução}

Previsto no art. 155, II, da Constituição Federal de 1988, o ICMS (Imposto sobre Circulação de Mercadorias e Prestação de Serviços) é responsável pela maior parte da arrecadação dos Estados. A cobrança de tributos é a principal fonte de receitas públicas, por meio das quais o estado deve cumprir sua finalidade redistributiva e atingir os objetivos fundamentais presentes no art. $3^{\circ}$ da Constituição Federal, dentre os quais a 
construção de uma sociedade livre, justa e solidária, a erradicação da pobreza, e a redução das desigualdades sociais e regionais [Viol 2005].

Desde a publicação da lei 6.474/2004, o Estado de Alagoas realiza a cobrança do imposto de antecipação do ICMS em função do diferencial de alíquota devido ao Estado nas compras realizadas em outros entes da federação. Tal imposto é calculado com base na origem dos produtos, sendo o valor cobrado do contribuinte Alagoano que esteja regular, em um intervalo de 20 a 80 dias da efetiva passagem da mercadoria por um posto fiscal de fronteira. Com o advento da Nota Fiscal Eletrônica (NF-e) e o aumento do número de informações disponibilizadas de forma eletrônica na nota fiscal, um novo momento de apuração de tributos passou a ser possível. Além da cobrança do diferencial de alíquota, o Estado de Alagoas pode realizar o acompanhamento dos valores de substituição tributária, verificando a efetividade do recolhimento destes tributos de forma a permitir um melhor aproveitamento das ações de fiscalização de trânsito.

Na classificação dos produtos, a Secretaria da Fazenda deve avaliar se os emitentes das notas fiscais estão preenchendo os campos da NF-e de forma correta, permitindo a cobrança e fiscalização dos impostos incidentes na operação de circulação de mercadorias entre a cadeia de distribuição (indústria, atacadistas e varejistas). Esta tarefa não é trivial, pois envolve as diversas variáveis constantes nas notas fiscais, além da prolífera e mutável legislação acerca do tema, o que implica em constantes mudanças nas regras de classificação. A complexidade cresce devido à necessidade de dados complementares, não constantes na nota fiscal, sejam oriundos de outras fontes de dados (Receita Federal do Brasil - RFB), ou produzidos pela própria Secretaria da Fazenda do Estado de Alagoas.

Nas últimas décadas, modelos baseados em regras foram muito utilizados no auxílio da administração tributária. Estas regras são definidas por especialistas, mapeando seu conhecimento do domínio para sistemas de decisão. No entanto, diante de um cenário onde há um volume crescente de dados tributários, a manutenção destes sistemas se torna custosa [Chen et al. 2018]. A inteligência artificial é uma importante aliada na administração tributária, permitindo encontrar padrões nos dados puros e resolver problemas que outrora necessitavam da participação humana. Exemplos de aplicações empregadas atualmente são a detecção de transações relacionadas a fraudes [Paula et al. 2016] e lavagem de dinheiro [Chen et al. 2018].

Neste contexto, este trabalho apresenta o Cálculo Automático de Tributos (CALT), uma ferramenta para que se obtenha, de forma automática e antecipada, o correto valor do ICMS a ser cobrado em operações interestaduais no Estado de Alagoas. Mediante o agrupamento de produtos similares a uma mesma classe tributária, pretende-se classificar novos produtos quanto a sua tributação de acordo com os campos especificados na nota fiscal. Ademais, o CALT implementa um procedimento de dupla-checagem que permite a evolução contínua do classificador bem como a detecção de indícios de acometimento de infrações.

A contribuição efetiva deste trabalho está na redução da fiscalização manual e facilidade do tratamento tributário das mercadorias em trânsito, permitindo ao Fisco Estadual um melhor controle e atuação na fiscalização. Os experimentos realizados mostraram que o CALT foi capaz de analisar cerca de 100 mil notas fiscais por dia, utilizando-se de uma infraestrutura de processamento de baixo custo, valor proibitivo para ser realizado 
de forma manual. Vale salientar que o calculo é feito no momento que a nota é emitida, como temos a entrada de 20 mil notas por dia em média, essa quantidade de notas avaliadas ocorreu apenas em casos de cálculos retroativos. Além disso, os resultados indicaram um aumento de cerca de $40 \%$ na arrecadação mensal em uma das classes tributárias avaliadas.Vale salientar que o registro do software em tela encontra-se depositado no INPI sob o número BR512020001196-9.

O restante deste trabalho está organizado em 5 seções. A seção 2 apresenta uma revisão sobre o cálculo tributário, destacando aspectos relacionados à cobrança automática e as técnicas mais utilizadas. A seção 3 apresenta uma visão geral da ferramenta CALT. Em seguida, a seção 3.2 descreve o processo de cálculo automático por meio do sistema de agrupamentos. Os experimentos que demonstram a viabilidade da ferramenta proposta neste trabalho são descritos na seção 4 . Por fim, a seção 5 apresenta as considerações finais deste trabalho.

\section{Trabalhos relacionados}

Um esforço considerável tem sido feito para combater a prática de fraude fiscal no Brasil. O uso de inteligência artificial na gestão tributária tem mostrado resultados promissores nas tarefas de fiscalização. Dentre as principais contribuições neste campo, destaca-se o aprimoramento da conferência tributária. Neste contexto, grande parte das abordagens presentes na literatura concentraram-se em propor modelos preditivos de classificação de contribuintes de ICMS [Galdino et al. 2004, Rocha 2017, Oliveira 2019].

A previsão da receita tributária é uma atividade fundamental para o planejamento e gestão eficiente do estado. Neste sentido, os autores [Carmo et al. 2019] aplicaram dois métodos para realizar a predição da arrecadação do ICMS no estado do Espírito Santo. O primeiro usa uma rede neural de múltiplas camadas com seleção de atributos em cascata, enquanto o segundo combina modelos tradicionais de previsão. Os métodos propostos foram eficazes em aperfeiçoar a previsão da dinâmica econômica do modelo de referência, onde foi possível obter uma melhora de até $16.2 \%$ no erro médio absoluto percentual simétrico (SMAPE).

Embora estes trabalhos sejam bem sucedidos ao utilizar inteligência computacional no âmbito fiscal, os autores não consideram a emissão de notas fiscais com informações incorretas. Neste sentido, os autores [Neto and Pará 2018] utilizaram métodos estatísticos e de mineração de dados para detectar indícios de sonegação fiscal. Eles utilizaram a Análise Envoltória de Dados (DEA) para identificar comportamentos suspeitos de empresas contribuintes. Em seguida, buscaram identificar anomalias na escrituração de notas fiscais emitidas pelas empresas suspeitas. Este trabalho mostrou que é possível proporcionar um melhor controle do risco de evasão tributária do ICMS a partir de um tratamento sistemático dos dados.

Outro trabalho, proposto por [Paula et al. 2016], utilizou um modelo de aprendizagem profunda para classificar exportadores de acordo com a possibilidade de cometimento de fraudes de exportação. Os autores aplicaram AutoEncoders em bases de dados de comércio exterior de bens e serviços em 2014 no Brasil para detectar situações que divergissem dos padrões. A análise por especialistas indicou que o modelo foi eficiente, identificando casos já conhecidos de fraudes. No entanto, não houve uma avaliação mais aprofundada dos resultados. 
Os autores [Madeira 2015] utilizaram técnicas de aprendizagem nãosupervisionada (K-Means) e supervisionada (Naive Bayes e Stochastic Gradient Descent) para a detecção de discrepâncias em Notas Fiscais de Serviço Eletrônicas (NFS-e) de código 07.19.04 (engenharia consultiva - acompanhamento e fiscalização da execução de obras de engenharia, arquitetura e urbanismo) no Município do Rio de Janeiro. Os modelos buscam separar as NFS-e em duas categorias: "tributadas no município" e "tributadas fora do município". As empresas com maior quantidade de notas classificadas incorretamente pelos modelos supervisionados foram selecionadas para a malha da fiscalização. A técnica indicou, das 768 empresas, 30 que se encaixavam nestes critérios. Das 30 empresas, a análise por parte do especialista indicou que 16 $(53,3 \%)$ realmente deveriam ser fiscalizadas.

Outros trabalhos na literatura buscaram aprimorar os procedimentos de conferência tributária a fim de garantir a escrituração correta dos documentos fiscais. Neste sentido, [Rocha 2018] utilizou a plataforma Watson e propôs uma solução para verificar se a alíquota do ICMS foi informada corretamente na nota fiscal. Para identificar a alíquota correta, os autores avaliaram algumas técnicas de classificação. O algoritmo Random Forest obteve cerca de $98 \%$ de assertividade, porém não conseguiu aprender os padrões de classificação para algumas categorias de produtos.

O Busca.Legal T2 é um assistente pessoal tributário que utiliza computação cognitiva para obter a tributação de um produto a partir da descrição, NCM ou código de barras desse produto [Busca.Legal 2020]. A ferramenta é capaz de identificar a tributação e realizar o cálculo dos tributos de operações internas e interestaduais entre as 27 unidades federativas. O Busca.Legal T2 considera todas as particularidades da legislação, porém necessita que o usuário forneça os dados manualmente para a realização do cálculo.

O sistema apresentado neste trabalho visa calcular os tributos devidos nas operações interestaduais de trânsito de mercadorias destinadas ao Estado de Alagoas, fazendo uso de um sistema de regras de classificação e técnicas de agrupamento. Diferente, por exemplo do Busca.Legal T2, as informações do cálculo são extraídas diretamente da NF-e, reduzindo a necessidade de intervenção humana para a realização do cálculo. Além disso, possui um mecanismo para realizar a constante evolução do sistema de classificação por meio da interação com o fiscal tributário.

\section{Cálculo Automático de Tributos (CALT)}

A classificação dos produtos é uma tarefa fundamental do cálculo do ICMS, por meio da qual os fiscais definem a modalidade de tributação mais adequada aos produtos de uma NF-e. A classificação é uma ação complexa, que envolve consultas a diversos sistemas para a busca de informações que subsidiem o fiscal no momento de calcular o imposto. Quando realizada de forma manual, demanda muito esforço e é suscetível a erros, ocasionando no subsequente erro de cálculo do imposto.

O CALT é fruto de uma iniciativa da Secretaria de Fazenda do Estado de Alagoas (SEFAZ-AL), em parceria com a Universidade Federal de Alagoas (UFAL) e a Fundação de Amparo à Pesquisa do Estado de Alagoas (FAPEAL). O CALT consiste em uma solução de cálculo automatizado dos tributos devidos nas operações interestaduais de trânsito de mercadorias destinadas ao estado de Alagoas. Com base nas informações presentes na nota fiscal, define-se a classe de tributação à qual os produtos presentes nesta 
nota devem pertencer. Para cada uma dessas classes, um determinado conjunto de decisões deve ser empregado no sentido de se obter o cálculo do imposto incidente (ver Figura 1).

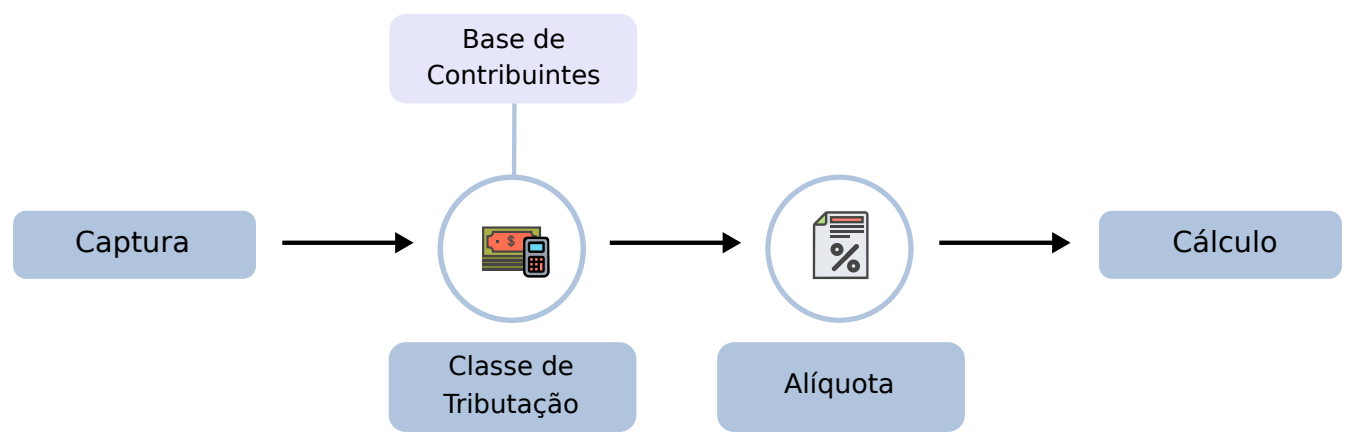

Figura 1. Etapas principais do cálculo do imposto.

Em termos gerais, o processo de cálculo automático de tributos envolve quatro etapas principais:

1. Captura dos documentos fiscais: Inicialmente, é realizada a captura das informações necessárias para a realização do cálculo. Para tal, o sistema realiza consultas periódicas às bases de dados da SEFAZ-AL, obtendo os dados das NF-e mais recentes, além de informações auxiliares que não constam na NF-e, como o cadastro de contribuintes do estado.

2. Definição da classe de tributação: Em seguida, define-se a classe de tributação utilizada na classificação dos produtos da referida NF-e. As três principais classes são: Diferencial de Alíquotas (DIFAL), Antecipado (ANT) e Substituição Tributária (ST). Há uma fórmula associada a cada classe, que junto com a alíquota, definem o valor do imposto a ser pago no produto.

3. Definição da alíquota: Nesta etapa, é feita a verificação dos parâmetros de classificação do produto para o cálculo tributário, por meio da definição das alíquotas interestaduais, redução da base da cálculo, análise dos casos de imunidade, isenção e situações especiais de cobrança, bem como a definição dos prazos e do responsável pelo pagamento.

4. Cálculo do valor: Por fim, com base nestas classificações é feito, então, o cálculo do imposto a partir da aplicação das fórmulas de cálculo para cada sistemática de tributação.

Para resolver o problema de classificação dos produtos da NF-e, duas abordagens complementares foram propostas. A primeira abordagem (Seção 3.1) consiste num sistema especialista baseado em regras que codificam o entendimento da legislação tributária, tomando proveito de uma arquitetura que desacopla o código do sistema das regras de classificação tributária. Ou seja, a criação e manutenção das regras pode ser feita sem a necessidade de alterações no código do sistema.

A segunda abordagem (Seção 3.2) é a análise de agrupamentos, que consiste no agrupamento de produtos com informações similares por meio de técnicas de aprendizagem não supervisionada. Em seguida, cabe a um agente identificar as características dos produtos e rotular os grupos com a classificação tributária que reflete aquele grupo. Esse agente pode ser um especialista treinado ou rotinas pré-programadas. Dessa forma, 
os itens da NF-e são atribuídos a um grupo, cujo rótulo reflete a sua rotulação por esse segundo sistema.

As seções seguintes descrevem detalhadamente as duas abordagens desenvolvidas para auxiliar na tarefa de classificação de produtos. Ademais, as duas abordagens podem cooperar entre si através do procedimento de dupla-checagem (Seção 3.3), que possibilita ao fiscal interagir com o sistema e tratar os casos de divergência entre os classificadores. Deste modo, o CALT implementa um mecanismo de retroalimentação em que é possível aprender com os erros e evoluir o modelo de classificação.

\subsection{Sistema Especialista}

O sistema especialista foi elaborado de forma a mapear o conhecimento dos fiscais com relação às leis de tributação em vigor. A classificação é feita realizando verificações de regras que modelam as decisões que seriam realizadas pelo fiscal. Partindo de um estado inicial, o classificador irá percorrer uma sequência de estados até calcular o valor do imposto. A transição de um estado para o próximo ocorre após o acionamento de uma determinada regra de classificação. Deste modo, cada estado constitui um conjunto de regras de classificação e realiza uma etapa do cálculo.

A Figura 2 ilustra o modelo lógico de como é definida a classe de tributação a partir das informações constantes na NF-e e no cadastro de contribuintes do estado registrados na SEFAZ. O estado inicial contempla a captura da nota fiscal e importação de campos relevantes para a classificação, como a data de emissão do documento fiscal, o tipo de operação e o estado emissor.

Em seguida, verificam-se as exceções de DIFAL, isto é, se a nota foi emitida em decorrência de vendas por marketing direto, casos de faturamento de veículos, venda de energia elétrica, dentro outros. O próximo estado irá verificar se o contribuinte é regularmente cadastrado no estado. Se não tiver um cadastro ativo, considera-se que todos os produtos desta nota sejam calculados a partir da sistemática DIFAL.

Caso o destinatário seja um contribuinte regular, são realizadas as verificações dos casos de ST constantes no convênio 52/91 e Antecipado, caso contrário. Por fim, verificase algumas exceções específicas, como por exemplo, se o produto é um ativo ou é para consumo e, portanto, utiliza-se o ST por Diferencial de Alíquotas.

Definida a sistemática de tributação, verificam-se os parâmetros de classificação do produto para o cálculo do tributo devido (ver Figura 3), por meio da definição das alíquotas interestaduais, redução da Base de Cálculo (BC), análise dos casos de imunidade, isenção e situações especiais de cobrança, bem como a definição dos prazos e do responsável pelo pagamento. Ao fim de cada verificação, caso todas as etapas tenham sido realizadas, o resultado do cálculo será armazenado na memória, que consiste do registro de decisão e dos parâmetros classificados nesses processos.

Caso tenha ocorrido algum erro nessas verificações, seja por critérios mal definidos ou dados incoerentes nas notas fiscais que impossibilitem a correta classificação, estes casos serão separados para posterior tratamento, seja na reavaliação dos processos ou na verificação dos erros de preenchimento presentes. Com base nessas classificações temos, por fim, o cálculo do imposto devido a partir da aplicação das fórmulas de cálculo para cada sistemática de tributação. 


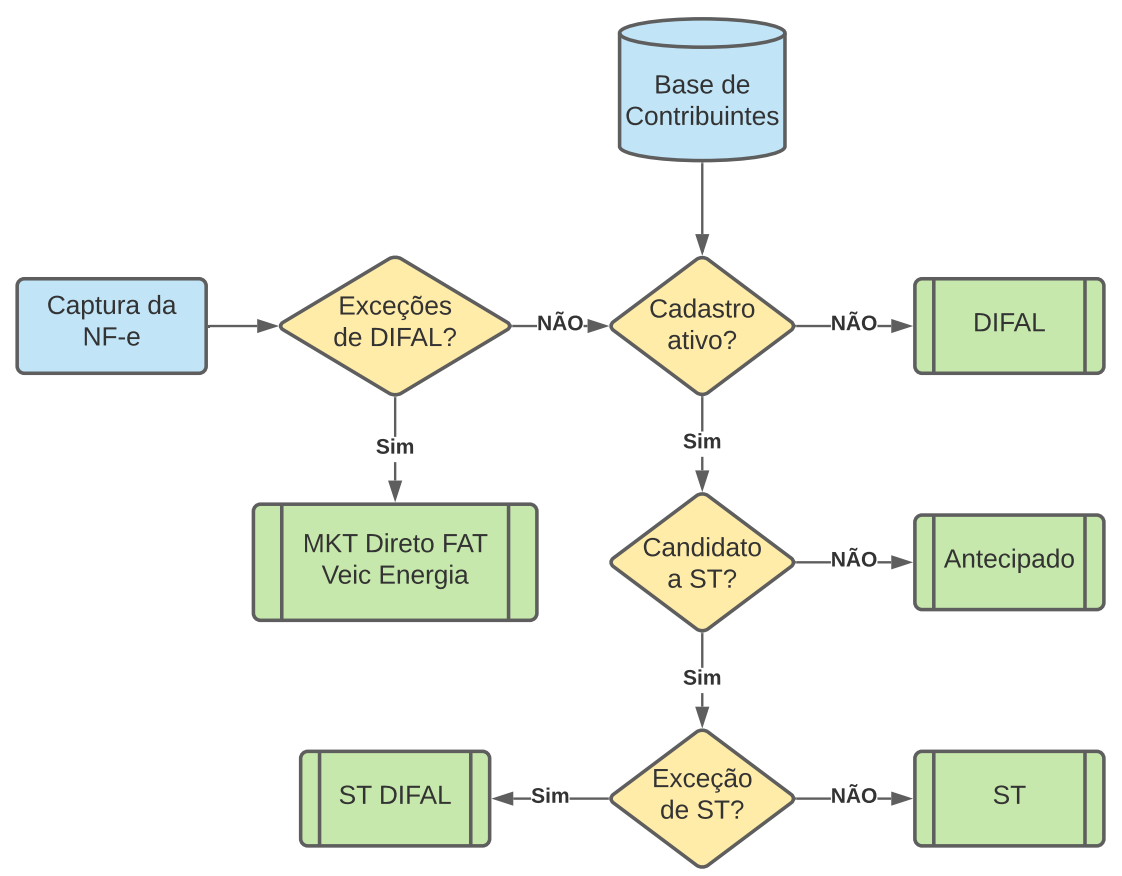

Figura 2. Etapas principais para a classificação tributária.

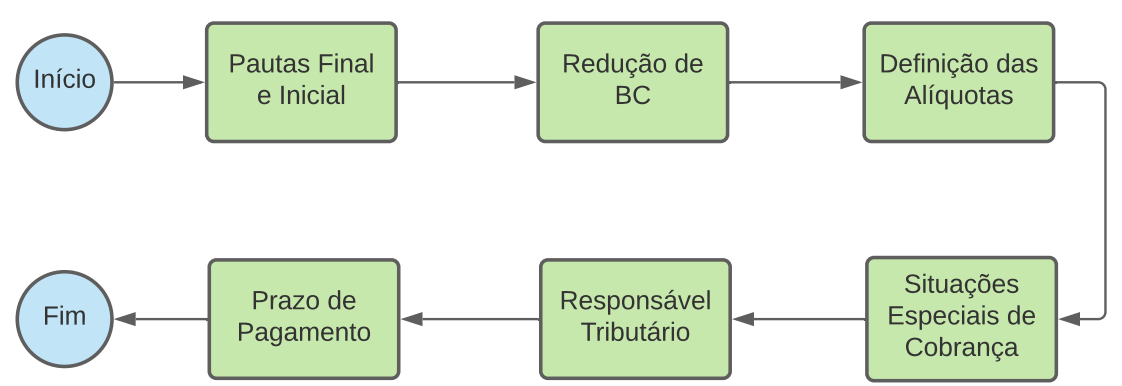

Figura 3. Etapas da identificação dos parâmetros de tributação e cálculo.

Todas as regras são criadas a partir de comparadores, que quando combinados produzem um valor booleano. Por exemplo: verifique se o tipo da nota é saída e a finalidade é normal. Dependendo do resultado dessas verificações, o fluxo seguirá de forma a direcionar a identificação do tipo do imposto. As regras são cadastradas de antemão e podem ser modificadas a qualquer momento a fim de acompanhar as mudanças na legislação ou atualizar o entendimento da tributação de um produto.

Além de garantir o acerto por meio de regras embasadas na legislação, o sistema especialista pode facilmente explicar como o cálculo foi realizado caso haja contestação. No entanto, os dados presentes nas notas fiscais podem ser imprecisos ou conter erros de preenchimento, assim, uma abordagem puramente baseada em regras pode apresentar erros na classificação caso o dado apresentado não corresponda à expectativa. 


\subsection{Análise de Agrupamentos}

Mediante o uso de técnicas de aprendizagem de máquina (AM), o CALT explora padrões encontrados nos dados para tentar agrupar produtos de classes tributárias similares e construir um modelo de classificação que permita ao fiscal obter, de forma automática e antecipada, o correto valor do tributo a ser cobrado, bem como indícios de acometimento de infrações. A Figura 4 apresenta um esquema geral da solução adotada no CALT.

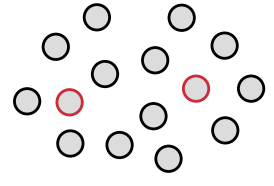

Centroides

(a) Treinamento.

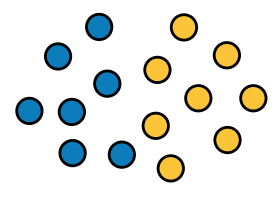

- Cluster \#1

○ Cluster \#2

(b) Agrupamento.

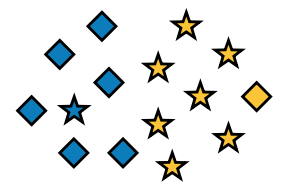

$\diamond$ Tipo ANT

t Tipo ST

(c) Rotulação.

Figura 4. Criação de grupos e rotulação.

Em um primeiro momento, é realizado o treinamento do modelo com base em um conjunto de NF-e, chegando a um conjunto de centroides que representam os grupos de itens - Figura 4(a). Em seguida, os itens são atribuídos aos seus centroides mais próximos, efetivamente criando os grupos - Figura 4(b). Por fim, tomando como base a classificação tributária gerada previamente pelo sistema especialista, ou por um grupo de auditores especialistas (em caso de se utilizar apenas a solução baseada em aprendizagem), é atribuído um rótulo ao grupo, conforme visto na Figura 4(c). No exemplo em questão, o Grupo \#1 é rotulado com a classificação tributária ANT, visto que esta é maioria dentre os itens desse grupo; de maneira análoga, o Grupo \#2 será rotulado com a classe tributária ST.

Os agrupamentos e rotulação serão utilizados para classificar os itens das NF-e que serão processadas a partir de então, conforme é apresentado na Figura 5.

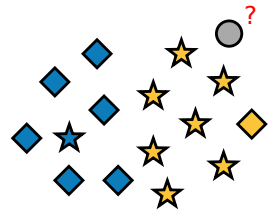

(a) Novo produto.

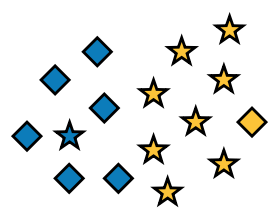

(b) Classificação.

Figura 5. Classificação majoritária.

Na Figura 5(a), um produto recém-chegado é comparado com os grupos existentes, verificando qual o centroide mais próximo. No caso do exemplo, o centroide do Grupo \#2. Na Figura 5(b), o produto em questão recebe o rótulo do Grupo \#2 (ST). 
As vantagens desse tipo de classificação está na exploração da imprecisão dos dados e automatização do processo por meio da AM. Enquanto que o sistema especialista carece de um mecanismo automatizado de aprendizagem, esta nova abordagem é mais flexível para as adaptações em outras realidades de tributação e robusta quanto a inconsistência nos dados de entrada.

\subsection{Procedimento de Dupla-checagem}

Quando há divergência na classificação obtida por meio do sistema especialista e baseado em agrupamentos, é gerado um alerta, permitindo ao fiscal decidir a classificação correta do produto. O processo é resumido na Figura 6.

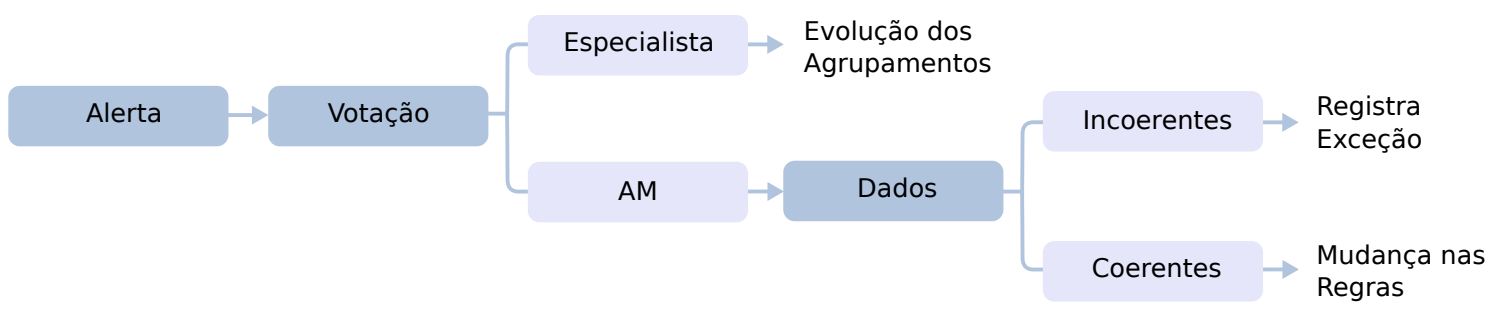

Figura 6. Processo de tratamento de anomalias.

Partindo do alerta, é apresentado ao fiscal um resumo daquela divergência, indicando a classificação de cada sistema e onde houve discordância entre as partes. Com base nos dados apresentados, o fiscal pode então votar em qual classificação é a correta, o que pode levar a ações corretivas:

1. Caso o sistema especialista seja indicado como correto, usa-se a informação para permitir que os agrupamentos sejam reorganizados de forma que itens similares ao que disparou o alerta sejam classificados corretamente no futuro. O reagrupamento é dado pela partição do grupo original em dois grupos, conforme apresentado na figura 7.

2. Caso o modelo AM seja indicado como correto, o erro do sistema especialista pode ser indício de duas possibilidades:

(a) Os dados da NF-e apresentam erros que levaram o sistema especialista a classificar os itens seguindo um fluxo incorreto de regras. Neste caso, é registrada uma exceção para que os fiscais tributários analisem o caso.

(b) As regras que geraram a saída em questão não estão corretas, sendo passíveis de mudanças.

Deste modo, ambos os sistemas estão em constante evolução de forma proativa, tomando sempre como base o feedback fornecido pelo especialista.

\section{Resultados e Discussão}

Devido ao grande volume de notas fiscais, a conferência sobre a corretude do imposto cobrado aos contribuintes pelos postos fiscais é realizada por meio de amostragem e demanda um grande esforço dos fiscais, que poderiam ser melhor alocados em atividades de fiscalização e cobrança. Em testes de desempenho, o sistema desenvolvido demonstrou a capacidade de analisar e calcular cerca de 500 mil notas fiscais - o equivalente à 


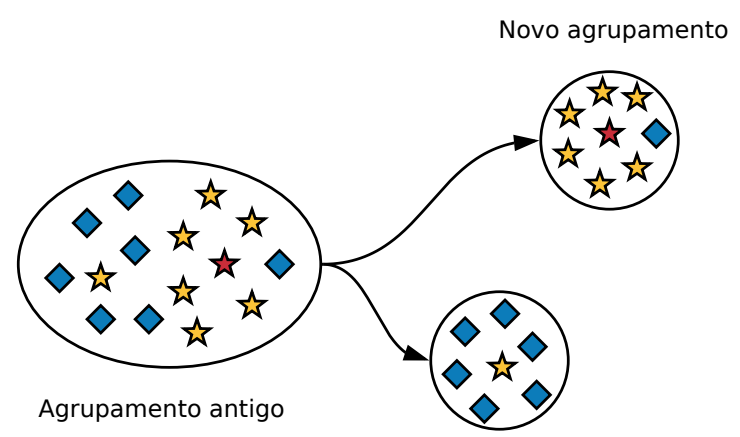

Figura 7. Evolução dos agrupamentos.

demanda média de um mês - em apenas 5 dias de processamento, utilizando-se de uma infraestrutura de baixo custo.

Como o processamento se dá de forma online, ou seja, à medida que as informações são acatadas na base de dados, o valor do tributo devido já é devidamente calculado e disponibilizado antes mesmo da chegada da mercadoria ao posto fiscal na fronteira do estado, salvo raras ocasiões de proximidade do emitente com o posto ou demora no acatamento das informações na base de dados.

A corretude da classificação foi atestada por meio da comparação entre a classificação definida pelos fiscais e pelo CALT. Para tal, foi selecionado o conjunto de NF-e paradas nos postos de fiscalização entre os meses de agosto a setembro de 2019, onde os fiscais realizaram o cálculo dos valores de tributo de forma manual. Estas notas correspondem a cerca de 5\% do fluxo de notas no período em questão. Nesta comparação, verificou-se que houve concordância entre os valores fornecidos pelo CALT e pelos fiscais em $98,2 \%$ dos casos analisados.

Desde maio de 2020, com a implantação do sistema unificado de cobrança do ICMS pelo Estado de Alagoas, os resultados do CALT começaram a ser utilizados de forma ampla nas operações interestaduais de mercadorias. De acordo com um levantamento realizado sobre a arrecadação do ICMS no Estado de Alagoas até abril 2021 [Júnior 2021], o primeiro quadrimestre de 2021 apresentou um aumento de $18 \%$ na arrecadação do ICMS em relação ao primeiro quadrimestre de 2020. Enquanto isso, nos primeiros quadrimestres dos anos anteriores, os aumentos variaram entre $1 \%$ a $6 \%$, o que indica um aumento acima da curva na arrecadação após a utilização do sistema. Vale salientar que o registro do software em tela encontra-se depositado no INPI sob o número BR512020001196-9.

\section{Conclusão}

A arrecadação do ICMS é uma das principais fontes de receita para os estados da federação, sendo de suma importância o seu cálculo, fiscalização e cobrança. No entanto, a intrincada legislação tributária e o grande volume de mercadorias em circulação impossibilita a realização dessas tarefas de forma manual.

Esse trabalho apresentou o CALT, uma solução que inova ao desacoplar a lógica de negócios do código do sistema, permitindo que os próprios fiscais tributários mantenham e atualizem as regras de negócio. A integração do CALT aos demais sistemas da 
SEFAZ-AL possibilitou ganhos em termos de agilidade dos processos, precisão e transparência das informações tributárias, além do grande potencial arrecadatório para o estado. Ademais, com a redução na carga de trabalho dos fiscais, pode-se alocar os esforços de trabalho para atividades de fiscalização e cobrança, constituindo um ganho de produtividade da secretaria como um todo.

Os próximos passos no desenvolvimento do CALT contemplam a implementação de soluções de ciência da dados e inteligência artificial às atividades tributárias, auxiliando na detecção de incoerências no preenchimento das notas fiscais, erros nas regras cadastradas pelos fiscais e extração automática de regras a partir do texto da lei.

\section{Agradecimentos}

Agradecemos à SEFAZ-AL e à FAPEAL pelo financiamento do projeto número 60030 000346/2017, que possibilitou o desenvolvimento do CALT.

\section{Referências}

Busca.Legal (2020). Busca.legal t2 - resolva suas dúvidas tributárias! [Acesso em 14 agosto 2020].

Carmo, M., Komati, K., and Boldt, F. (2019). Previsão de receitas de icms do estado do espírito santo através de seleção de características em cascata e técnicas de aprendizado de máquina. In Anais do XVI Encontro Nacional de Inteligência Artificial e Computacional, pages 118-129, Porto Alegre, RS, Brasil.

Chen, Z., Teoh, E. N., Nazir, A., Karuppiah, E. K., Lam, K. S., et al. (2018). Machine learning techniques for anti-money laundering (aml) solutions in suspicious transaction detection: a review. Knowledge and Information Systems, 57(2):245-285.

Galdino, G., Renato, M., and Fonseca Neto, R. (2004). Uma rede neural artificial de múltiplas camadas aplicadas ao combate à sonegação fiscal de icms. In Anais do XXXVI Simpósio Brasileiro de Pesquisa Operacional (SBPO).

Júnior, E. (2021). ICMS tem alta fora da curva e dispara em alagoas. http://edivaldojunior.blogsdagazetaweb.com/2021/05/10/ icms-tem-alta-fora-da-curva-e-dispara-em-alagoas/. [Online; acessado em 18 de maio de 2021].

Madeira, R. d. O. C. (2015). Aplicação de técnicas de mineração de texto na detecção de discrepâncias em documentos fiscais. $\mathrm{PhD}$ thesis, Fundação Getúlio Vargas.

Neto, B. and Pará, S. A. (2018). Análise de "outliers" para o controle do risco de evasão tributária do icms. Master's thesis, Universidade de Brasília.

Oliveira, V. D. (2019). Redes neurais artificiais aplicadas à identificação de riscos de inadimplência fiscal de icms e iss no distrito federal. Master's thesis, Universidade de Brasília.

Paula, E. L., Ladeira, M., Carvalho, R. N., and Marzagão, T. (2016). Deep learning anomaly detection as support fraud investigation in brazilian exports and anti-money laundering. In 2016 15th IEEE International Conference on Machine Learning and Applications (ICMLA), pages 954-960. IEEE. 
Rocha, L. F. d. (2018). Aprimorando conferências tributárias através da computação cognitiva utilizando a Plataforma Watson. Artigo de conclusão de curso (Bacharel em Ciência da Computação), Universidade da Passo Fundo, Passo Fundo, RS.

Rocha, S. M. (2017). Mineração de dados aplicada à classificação dos contribuintes de icms da sefaz-go. Master's thesis, Pontifícia Universidade Católica de Goiás.

Viol, A. L. (2005). A finalidade da tributação e sua difusão na sociedade. Seminário de Políticas Tributárias, 2. 